\title{
Bifurcation properties of the average activity of interconnected neural populations
}

\author{
Arnaud Tonnelier \\ Techniques de l'Imagerie, de la Modélisation et de la Cognition, CNRS UMR 5525, Faculté de Médecine, F-38706 La Tronche, France \\ Laboratory of Computational Neuroscience, EPFL, CH-1015, Lausanne, Switzerland \\ Received: 21 May 2002 / Accepted: 25 April 2003 / Published online: 7 July 2003
}

\begin{abstract}
The relevant scale for the study of the electrical activity of neural networks is a problem of mathematical and biological interest. From a continuous model of the cortex activity we derive a simple model of an interconnected pair of excitatory and inhibitory neural populations that describes the activity of a homogeneous network. Our model depends on three parameters that stand for the scale variability of the network. A bifurcation analysis reveals a great variety of patterns that arise from the interplay of excitatory and inhibitory populations provided by synaptic interactions. We emphasize the differences between the dynamical regimes when considering a moderate and a high inhibitory scale. We discuss the consequences on a propagating activity.
\end{abstract}

\section{Introduction}

Mathematical models in neurophysiology use ordinary differential equations (ODE) to describe the behavior of an isolated neuron or a network of synaptically coupled neurons. The model developed by Hodgkin and Huxley (1952) is an example of such an ODE system that is widely used and studied. Using their formalism, a large number of models have been developed that are based on the description of specific electrical currents in cellular structures (Bower and Beeman 1994) or on a mathematical simplification of complete systems (e.g., FitzHugh 1961; Rinzel 1985). In order to analyze biological measurements such as the mapping of the cortical activity through optical recording and to understand the functions of large neuron assemblies, we are interested in the role of the spatial scale in modeling neural networks. The construction of a neural network depends on (i) the characteristics of the

Correspondence to: A. Tonnelier (e-mail: Arnaud.Tonnelier@epfl.ch) individual neurons and (ii) the network architecture, i.e., the synaptic interactions. The dynamical behavior of the network is specified either by an ODE system for each neuron or by an equation for a neural population without any reference to the complex behavior of a single neuron. For neural networks made of a large number of neurons, the first approach leads to a complex ODE system that appears to be mathematically intractable. The second method reduces the complexity of the problem, but the equations for a neural assembly are difficult to derive because of the intrinsic nonlinearity of the dynamics.

In this paper, we study a neural network whose kinetics are described by a simplified model motivated by biological data from the piriform cortex (Haberly and Price 1978; Ballain et al. 1998). The neuronal density incites us to describe neuron assemblies through a continuous representation. In addition, the connections are given by a continuous mapping from one point of the network to an other. Using this approach, the problem is to determine the relevant scale at which the network presents a complexity of the dynamics at an intermediate stage between the activity of a single neuron, on the one hand, and the activity of the total neuronal assembly, on the other. Moreover, without restrictive assumptions on the connection strengths, it is difficult to obtain interesting properties on the dynamical behavior of the neural network. To get around these problems, we study subfields that present a synchronous activity. Motivation for this approach comes from physiological observations showing rhythmic organization for a large subpart of the network, although single neurons may fire asynchronously. This consideration leads to a simple model of excitatoryinhibitory populations described by a set of two nonlinear ODEs that does not require a precise description of the network connectivity. Our model may be seen as a macroscopic description with a characteristic scale, which determines the relevance of neuronal activity for the biological function of the network.

Macroscopic models are useful in studying collective behavior of cell assemblies like macroscopic oscillations. Pioneering work in developing macroscopic models was 
performed by Amari (1971). The model that we derived is a neural oscillator, which presents some features similar to macroscopic models already studied (Wilson and Cowan 1972; Borisyuk and Kirillov 1992). It is known that neural networks composed of coupled neural oscillators present synchronous oscillatory activities (Destexhe 1994; Tonnelier et al. 1999). It is argued that oscillatory modes may be involved in information coding (Atiya and Baldi 1989; Chang at al. 1992) or in information processing (Erdi et al. 1993). Using the bifurcation theory, we study how oscillatory activities emerge from the interplay of excitatory and inhibitory populations provided by synaptic connections. Our analysis is a first step in the understanding of complex spatiotemporal behavior in the low-level network consisting of interactive excitatory and inhibitory neurons (Destexhe 1994).

The paper is organized as follows. In Sect. 2, a mathematical model of our cortical neural network is presented. The third section is devoted to the bifurcation analysis of the synchronous activity and the classification of all possible regimes and transitions between them. The scale-parameter space is partitioned into domains of equivalent behavior bounded by bifurcation curves. Next, we study a neural network made of neural oscillators coupled through feedforward connections. We are interested in oscillatory activity and its propagation driven by synaptic modifications.

\section{The model}

Neurons receive and emit action potentials. We focus solely on the synaptic transfer of information from one neuron to another and ignore chemical or other information transfer processes. A synaptic current without delay or dendritic dependences has the form (see, for example, Abbott and Marder 1989):

$I_{s y n}=\bar{g} s(t)\left(V_{p o s t}-V_{e q}\right)$

where $V_{e q}$ is the synaptic reversal potential, $V_{p o s t}$ is the potential of the postsynaptic neuron, and $\bar{g}$ is the maximal conductance of the synaptic current. The variable $s$ is a dynamic variable in the range $0 \leq s \leq 1$; it is the probability that the corresponding channel is in an open conducting state. In general, $s$ is a function of the presynaptic potential, $V_{\text {pre }}$. A number of different equations are used to model the function $s\left(V_{p r e}\right)$; it is either of a predefined form or satisfies a differential equation. For simplicity, we use a sigmoid-type function $s(t)=\Phi\left(V_{\text {pre }}(t)\right)$ where $\Phi: R \rightarrow[0,1]$ is a bounded and increasing function. The coupling of two neurons results in the equation for the postsynaptic neuron:

$\frac{\mathrm{dV}_{\text {post }}}{\mathrm{dt}}=r\left(V_{\text {post }}\right)(t)-\bar{g} \Phi\left(V_{\text {pre }}(t)\right)\left(V_{\text {post }}(t)-V_{\text {eq }}\right)$

where $r\left(V_{\text {post }}\right)$ gives the behavior of an isolated neuron. In the present work, we adopt a simple relaxation dynamics, i.e., $r\left(V_{\text {post }}\right)=-V_{\text {post }} / \tau$, where $\tau$ is the relaxation time of the neuronal membrane.
We consider two populations of neurons, one excitatory and the other inhibitory. Let us denote $v(x, t)$ $(u(x, t))$, the membrane potential of an excitatory (inhibitory) neuron located at $x \in \Omega_{e}\left(x \in \Omega_{i}\right)$ at time $t$, where $\Omega_{e} \subset R^{3}\left(\Omega_{i} \subset R^{3}\right)$, is the support of the excitatory (inhibitory) neural population. We note $\Omega=\Omega_{e} \cup \Omega_{i}$ the total network. One may interpret $\Phi(v(x, t))$ and $\Phi(u(x, t))$ as the instantaneous firing rate associated with the excitatory and the inhibitory population, respectively. The excitatory neurons receive three kinds of inputs: (i) a direct external sensory input, denoted by $F$; (ii) excitations from other neurons in $\Omega_{e}$; and (iii) inhibitory inputs from neurons in $\Omega_{i}$; conversely, inhibitory neurons are excited by neurons in $\Omega_{e}$ only. This situation mimics the organization of the cortex, particularly the rat olfactory cortex, which involves two kinds of cell populations: the pyramidal cells and the inhibitory interneurons (Haberly and Price 1978). However, our equations can describe other situations where such loops are involved in the regulation of the neural activity.

Since there is a continuum of neurons in the two domains $\Omega_{e}$ and $\Omega_{i}$, the membrane potential of a cell at position $x \in \Omega_{e} \cup \Omega_{i}$ depends on the synaptic influence of other cells in the network. A spatial conductance function $g(y, x)$ allows us to describe the connectivity from a cell at position $y$ to a cell at position $x$. The synaptic current due to the excitatory-excitatory interaction is given by

$$
\begin{aligned}
& I_{\text {Syn }}(x, t) \\
& \quad=\left(v(x, t)-v_{e q}\right) \int_{\Omega_{e}} g(y, x) \Phi(v(y, t)) \mathrm{d} y, \quad \text { for } \mathrm{x} \in \Omega_{\mathrm{e}}
\end{aligned}
$$

This synaptic current is generalized to the other interactions. The equations governing the time evolution are specified by a set of coupled nonlinear integrodifferential equations, namely:

$$
\begin{aligned}
\frac{\partial v}{\partial t}(x, t)= & -v(x, t)+F(x, t)\left(u_{e q}-v(x, t)\right) \\
& +\left(u_{e q}^{e e}-v(x, t)\right) \int_{\Omega_{e}} g(y, x) \Phi(v(y, t)) \mathrm{d} y \\
& +\left(u_{e q}^{i e}-v(x, t)\right) \int_{\Omega_{i}} g(y, x) \Phi(u(y, t)) \mathrm{d} y \\
\frac{\partial u}{\partial t}(x, t)= & -u(x, t) \\
& +\left(u_{e q}^{e i}-u(x, t)\right) \int_{\Omega_{e}} g(y, x) \Phi(v(y, t)) \mathrm{d} y
\end{aligned}
$$

where $F(x, t)$ is the external input on $x$ at time $t$, $g: \Omega \times \Omega \rightarrow R^{+}$is the positive conductance function giving the synaptic input weight from $y$ to $x$, and $t$ represents a reduced dimensionless time defined as $\tilde{t} / \tau$ where $\tilde{t}$ is the actual time. The equilibrium potentials are parameters that satisfy: $u_{e q}=u_{e q}^{e e}=u_{e q}^{e i}>0, u_{e q}^{i e}<0$ and $\left|u_{e q}^{i e}\right|<u_{e q}^{e e}$. Since we assume that inhibitory neurons 
have synaptic connections with excitatory neurons only, we have $g(y, x)=0$ for $(y, x) \in \Omega_{i}^{2}$. One may remark that the nonlinearity arises from (i) the function $\Phi$ and (ii) its product with $u_{e q}-v\left(\right.$ or $\left.u_{e q}-u\right)$.

\section{Synchronous activity}

\subsection{Definition of the problem}

We study the dynamics in absence of external stimulation, i.e., $F(x, t)=0$. We address the question of how the dynamical behavior of the network is affected by the conductance function $g$. In the first step, we consider regions in the network that exhibit a synchronous activity. Therefore, we look for spatially homogeneous solutions where the neuronal activity does not depend on space, and we note $v(t)(u(t))$ the excitatory (inhibitory) activity. Moreover, we assume that $\int_{\Omega} g(y, x) d y$ does not depend on $x$ (for $x \in \Omega_{e}$ or $\left.x \in \Omega_{i}\right)$. This choice includes the usual interaction function where the conductance $g(y, x)$ depends only on the difference $y-x$ :

$g(y, x)=w(y-x)$

where $w$ is a continuous, bounded, and positive function. Then (Eq. 1) becomes:

$$
\begin{aligned}
v^{\prime}(t)= & -v(t)+c_{e e} \Phi(v(t))\left(u_{e q}^{e e}-v(t)\right) \\
& +c_{i e} \Phi(u(t))\left(u_{e q}^{i e}-v(t)\right) \\
u^{\prime}(t)= & -u(t)+c_{e i} \Phi(v(t))\left(u_{e q}^{e i}-u(t)\right)
\end{aligned}
$$

where:

$$
\begin{gathered}
c_{e e}=\int_{\Omega_{e}} g(y, x) d y \text { for } x \in \Omega_{e}, \\
c_{i e}=\int_{\Omega_{i}} g(y, x) d y \text { for } x \in \Omega_{e}, \\
c_{e i}=\int_{\Omega_{e}} g(y, x) d y \text { for } x \in \Omega_{i} .
\end{gathered}
$$

The above transformation, yielding Eq. 2, allows one to reduce the infinite dimensional parameter space, $g \in L^{\infty}(\Omega \times \Omega)$, to a three-dimensional parameter space, $\left(c_{e e}, c_{i e}, c_{e i}\right) \in R^{3}$. Under this weak assumption on $g$, our study does not impose a specific connectivity of the network. Since $g$ is positive, $\left(c_{e e}, c_{i e}, c_{e i}\right)$ are positive numbers and the nature of the connections are given by the sign of $u_{e q}$ (Fig. 1). In fact, we will show that $v>u_{e q}^{i e}$ (see proposition 1), and therefore the term $c_{i e}\left(u_{e q}^{i e}-v\right)$, has an inhibitory effect modulated by $\Phi(u)$.

The two autonomous, nonlinear, first-order differential equations (Eq. 2) describe the synchronous activity of excitatory and inhibitory neural populations and are similar to, but different from, the Wilson and Cowan model (1972). The coefficients $c_{e e}, c_{i e}, c_{e i}$ synthesize the synaptic architecture, and their values are proportional to the size of the synchronous region, i.e., the number of cells involved in the synchronous activity. We have $c_{e e} \leq \operatorname{mes}\left(\Omega_{e}\right)\left\|g_{e e}\right\|_{L^{\infty}}$ (and similar expressions for $c_{i e}$ and $c_{e i}$ ) where mes $(\Omega)$ is the measure of $\Omega$. If we normalize these coefficients such that $c_{e e}=1$, for an arbitrarily chosen domain of reference $\left(\Omega_{e}\right)_{r e f}$, then the

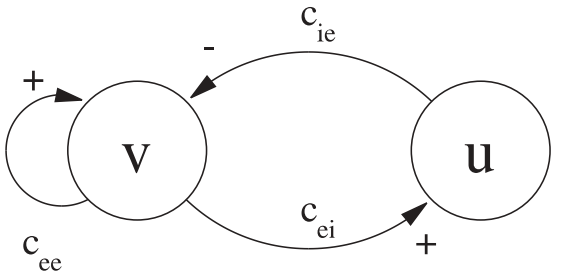

Fig. 1. Scheme of connections of the excitatory-inhibitory macroscopic model (Eq. 2). $v(u)$ is the activity of the excitatory (inhibitory) neural assembly. Parameters $\left(c_{e e}, c_{i e}, c_{e i}\right)$ are the weights related to the connections between populations and depend on the scale of interactions

relative difference of volume between $\left(\Omega_{e}\right)_{\text {ref }}$ and $\left(\Omega_{e}\right)$ affects in the same manner the value of $c_{e e}$. Therefore, these coefficients represent the scale of the neuronal interactions rather than the individual synaptic connection weights. For $c_{i e}=0$ and $c_{e i}=0$, each population acts as an isolated system that exhibits a trivial behavior. As $c_{i e}$ and $c_{e i}$ increase, different complex synchronous activities emerge depending on the value of $c_{e e}$. In the following, we analyze how the behavior is affected by these connections and therefore by the relative depth of interactions.

\subsection{Fixed points and linear stability analysis}

We start with simple results on the activities $(v, u)$ and give some general properties of the excitatory and inhibitory dynamics. Let us denote $c_{x}$ as a representation of any of these scales.

Proposition 1 If $c_{x}=o(1)$, the activity is proportional to $c_{x}$. If $c_{x} \gg 1$, the excitatory (inhibitory) activity is proportional to $u_{e q}^{e e}\left(u_{e q}^{e i}\right.$, respectively). Otherwise, the activity is bounded by these values.

We shall sketch the proof of this property. The two nullclines, $N L_{e}, N L_{i}$, associated with $v$ and $u$, respectively, are given by:

$$
\begin{cases}N L_{i}: & u=\frac{c_{e i} u_{e q}^{e i} \Phi(v)}{1+c_{e i} \Phi(v)} \\ N L_{e}: & u=\Phi^{-1}\left(\frac{v-c_{e e} \phi(v)\left(u_{e q}^{e e}-v\right)}{c_{i e}\left(u_{e q}^{i e}-v\right)}\right)\end{cases}
$$

Let us define the rectangular domain $D \in R^{2}$ as:

$D=\left[\frac{c_{i e} u_{e q}^{i e}}{1+c_{i e}}, \frac{c_{e e} u_{e q}^{e e}}{1+c_{e e}}\right] \times\left[0, \frac{c_{e i} u_{e q}^{e i}}{1+c_{e i}}\right]$

From the equations of the nullclines, the vector field on $\partial D$ is directed inward. Therefore, $D$ is an invariant set of Eq. 2 and it is easy to show that $D$ is globally attractive. Moreover one has $u_{e q}^{e e} c_{e e} /\left(1+c_{e e}\right)=u_{e q}^{e e} c_{e e}+o\left(c_{e e}\right)$ and $u_{e q}^{e e} c_{e e} /\left(1+c_{e e}\right)=u_{e q}^{e e}+o\left(1 / c_{e e}\right)$. Similar expressions are obtained for $c_{e i}$ and $c_{i e}$. We have the following: 
Proposition 2 For all $c_{x}$, the network admits an odd number of nondegenerate fixed points. One fixed point out of two is a saddle node.

A fixed point $\left(v_{0}, u_{0}\right)$ is given by the intersection of the nullclines leading to the resolution of $l(v)=0$, where $l(v)=-v+c_{e e} \Phi(v)\left(u_{e q}^{e e}-v\right)+c_{i e} \Phi\left(u_{e q}^{e i} c_{e i} \Phi(v) /\left(1+c_{e i} \Phi\right.\right.$ $(v)))\left(u_{e q}^{i e}-v\right)$. This equation admits at least one real solution since $l$ is continuous and $\lim _{x \rightarrow-\infty} l(x)=+\infty$, $\lim _{x \rightarrow+\infty} l(x)=-\infty$. Let $J$ be the Jacobian matrix of Eq. 2 evaluated at $\left(v_{0}, u_{0}\right)$. We have:

$$
\begin{aligned}
\operatorname{det}(J)= & -l^{\prime}\left(v_{0}\right)\left(1+c_{e i} \Phi\left(v_{0}\right)\right), \\
\operatorname{tr}(J)= & -2-\left(c_{e i}+c_{e e}\right) \Phi\left(v_{0}\right)-c_{i e} \Phi\left(u_{0}\right) \\
& +c_{e e}\left(u_{e q}^{e e}-v_{0}\right) \Phi^{\prime}\left(v_{0}\right)
\end{aligned}
$$

The sign of $\operatorname{det}(J)$ changes from one isolated zero (of $l(v)$ ) to the next one (if $\operatorname{det}(J) \neq 0$ ). Therefore, two nonsaddle and nondegenerate fixed points (i.e., $\operatorname{det}(J)>0$ ) are separated by a saddle one (i.e., where $\operatorname{det}(J)<0)$.

\subsection{Analytical results for a simplified case}

To go further in the analysis of Eq. 2, we take $\Phi$ as the piecewise linear function defined by:

$\Phi(x)=\left\{\begin{array}{lc}0 & \text { if } x<u_{t h}-\frac{1}{2 \lambda} \\ 1 & \text { if } x>u_{t h}+\frac{1}{2 \lambda} \\ \lambda\left(x-u_{t h}\right)+\frac{1}{2} & \text { elsewhere }\end{array}\right.$

where the parameter $\lambda$ is the slope of $\Phi$ and $u_{t h}$ is the threshold. We define $u_{\min }=u_{t h}-1 /(2 \lambda)$ and $u_{\max }=u_{t h}+1 /(2 \lambda)$ and $\lambda$ such that $u_{\min }>0$ and $u_{\max }<u_{e q}^{e e}$. It should be noted that $u_{t h}$ is not a free parameter since a shift of $u_{e q}^{e e}, u_{e q}^{i e}$, and $u_{e q}^{e i}$ allows us to take $u_{t h}=0$, but in order to keep the biological significance of the problem, we do not make this choice. The analysis carried out in the appendix is summarized in the following proposition:

Proposition 3 Given the piecewise linear function $\Phi$ (Eq. 3), the point $(0,0)$ is a stable node. If $c_{e e}<u_{\max } /$ $u_{e e}-u_{\max },(0,0)$ is globally attractive. Otherwise, we distinguish:

- if $c_{e i}<u_{\min } /\left(u_{e i}-u_{\min }\right)=c_{e i, 1}$, then the only bifurcation that appears is a saddle node bifurcation when $c_{e e}=u_{\max } /\left(u_{e e}-u_{\max }\right)$ (a saddle for $v<u_{\max }$ and $a$ stable node for $\left.v>u_{\max }\right)$.

- if $c_{e i}>u_{\min } /\left(u_{e i}-u_{\min }\right)$, then the system admits two or four nondegenerate fixed points different from $(0,0)$.

The ones that satisfy $u_{\min }<v<u_{\max }$ and $u<u_{\min }$ or $u>u_{\max }$ are saddle fixed points. For $c_{e i}>u_{\max } /$ $\left(u_{e i}-u_{\max }\right)=c_{e i, 2}$, the one such that $v>u_{\max }$ is a stable node and the saddle node bifurcation equation is: $\left(c_{e e} u_{e q}^{e e}+c_{i e} u_{e q}^{i e}\right) /\left(1+c_{e e}+c_{i e}\right)=u_{\max }$.

Figure 2 is the illustration of the saddle node bifurcation obtained for $c_{e i}>c_{e i, 2}$. Proposition 3 will be useful in the
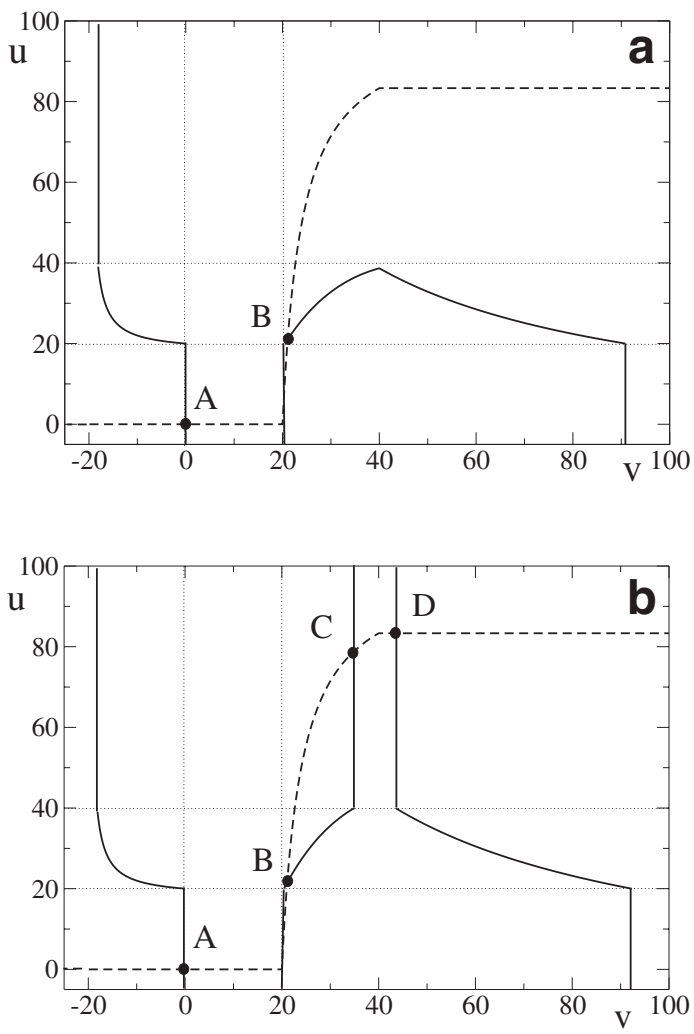

Fig. 2. Sketch of the nullclines of (Eq. 2) for $c_{e i} u_{e q}^{e i} /\left(1+c_{e i}\right)>u_{\max }$. The parameters are such that: $\left(c_{e e} u_{e q}^{e e}+c_{i e} u_{e q}^{i e}\right) /\left(1+c_{e e}+c_{i e}\right)<u_{\max }$ (Fig. 2a) and $\left(c_{e e} u_{e q}^{e e}+c_{i e} u_{e q}^{i e}\right) /\left(1+c_{e e}+c_{i e}\right)>u_{\max }$ (Fig. 2b). The dotted lines represent the boundaries between the rectangular domains where the study was carried out. The fixed points (labeled A, B, C, and $\mathrm{D}$ ) are indicated by black circles. The fixed points $\mathrm{C}$ and $\mathrm{D}$ appear through a saddle-node bifurcation

study carried out in the next section. First, it shows that the reversal potentials and the two potentials $u_{\min }, u_{\max }$ weighted by the connection parameters monitor the location of the bifurcations. Moreover, it emphasizes some specific ranges for the connection parameters. In particular, we find two characteristic values for $c_{e i}: c_{e i, 1}$ and $c_{e i, 2}$, which give the possible bifurcations that occur in our model. For instance, if $c_{e i}<c_{e i, 1}$, only one bifurcation occurs and no interesting dynamics emerges from the interplay of excitatory and inhibitory interactions. If $u_{e q}^{e e}=u_{e q}^{e i}$ and $\lambda$ is strong enough, then bifurcations appear when $c_{e e}$ and $c_{i e}$ are proportional to $u_{t h} /\left(u_{e q}^{e e}-u_{t h}\right)$. It reveals that a nontrivial behavior is expected when the strenghts of the excitatory and inhibitory interactions are close. We will elaborate this point in the following section.

\subsection{Bifurcation analysis for a regular function $\Phi$}

In this part, we study Eq. 2 in the regular case where $\Phi$ is the sigmoid function:

$\Phi(x)=\left(1+e^{-\lambda\left(x-u_{t h}\right)}\right)^{-1}$

where $u_{t h}=30$ and $\lambda=0.2$. We describe the various phase portraits of Eq. 2 as the parameters $\left(c_{e e}, c_{i e}, c_{e i}\right)$ 

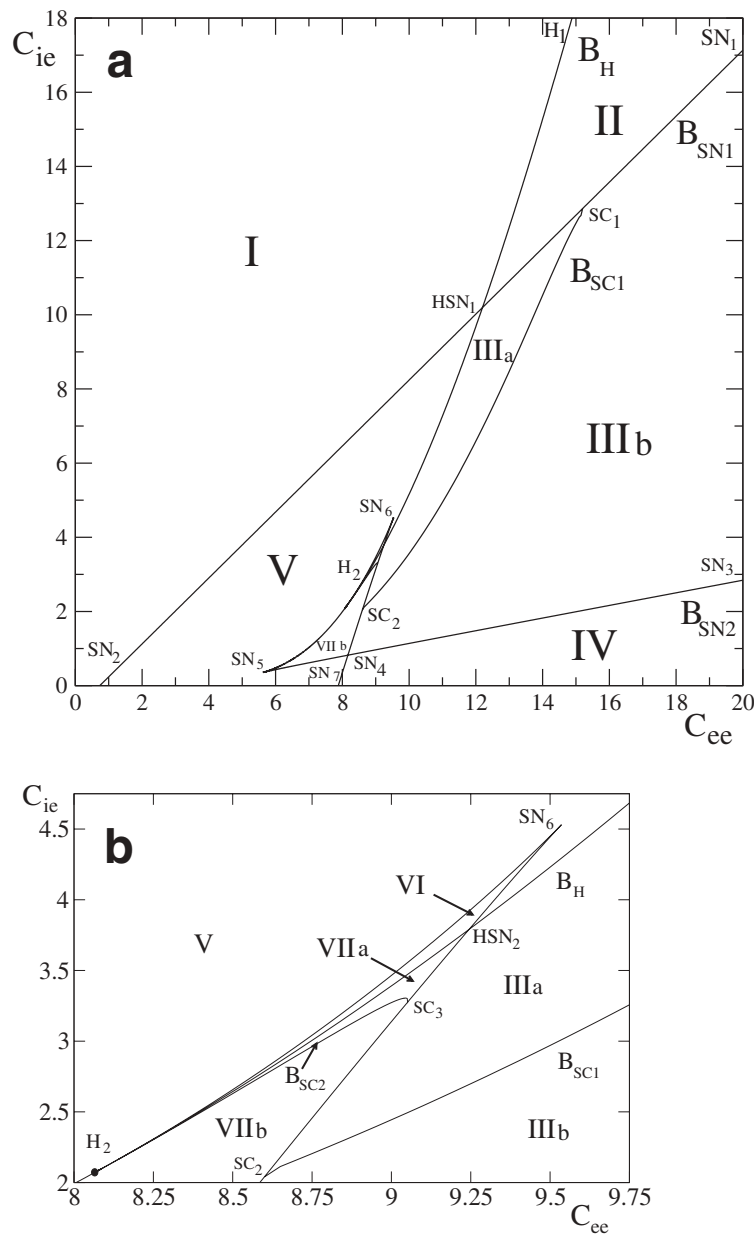

Fig. 3. The bifurcation diagram of (Eq. 2) for $c_{e i}=10$. This value satisfies: $c_{e i}>u_{\max } /\left(u_{e q}^{e i}-u_{\max }\right)$, which corresponds to one of the cases of proposition 3 . The other parameters are: $u_{e q}^{e e}=u_{e q}^{e i}=100$; $u_{e q}^{i e}=-20$. Figure $3 \mathrm{~b}$ is an enlargement of Fig. 3a

are changing. This allows us to predict qualitatively the behavior of the model in domains bounded by bifurcation curves. The previous study made useful the distinction between $c_{e i}$ greater or less than $u_{\max } /$ $\left(u_{e q}^{e i}-u_{\max }\right) \quad(\sim 0.48)$. Then we focus on two specific values of $c_{e i}$ that we report as a moderate and a high inhibitory scale, respectively. Our analysis does not capture the codimension three bifurcations that appear as $c_{e i}$ continuously increases. We will discuss this point further. In Figs. 3 and 5, we show the corresponding bifurcation curves in the plane $\left(c_{e e}, c_{i e}\right)$ for the two conditions specified above. In Figs. 4 and 6, we illustrate the representative phase diagrams for some relevant domains of Fig. 3 and Fig. 5, respectively. For details on the mathematical definitions, the reader is referred to Guckenheimer and Holmes (1983) and Kuznetsov (1995). The computation of local bifurcations has been carried out using the software program AUTO (Doedel 1981). Computation of global bifurcations were performed using a specific algorithm: the continuation of homoclinic orbit are obtained by computing the split function. Other numerical results were performed using the classical fourth-order Runge-Kutta method.
First, we comment on Fig. 3. We consider nine domains that define a partition of the $\left(c_{e e}, c_{i e}\right)$ plane where the dynamical system presents a structural stability. First we note that, (i) in domains I, II, and IV, we have a single fixed point (a sink in I and IV and a source in II); (ii) in domain III ( = IIIa $\cup$ IIIb), a source, a saddle, and a sink; (iii) in domain $\mathrm{V}$, two sinks and a saddle; (iv) in domain VI, three sinks and two saddles; and (v) in domain VII ( = VIIa $\cup$ VIIb), two sinks, two saddles, and a source. We list the bifurcations obtained from the numerical analysis. The curve $B_{X}$ gives the exact condition of bifurcation of type $X$, and $X_{i}$ stands for a point, indexed by an integer $i$, related to a bifurcation of type $X$ :

1. On the curve $\left(S N_{1}, S N_{2}\right)$, denoted by $B_{S N 1}$, and on the curve $\left(S N_{3}, \ldots, S N_{7}\right)$, denoted by $B_{S N 2}$, a saddle node bifurcation occurs. These saddle node bifurcations correspond to the coalescence and the disappearance of two equilibria as one crosses (i) from domain $\mathrm{V}$ to domains I or IV or (ii) from domain III to domains II or IV, and (iii) from domains VI or VII to domains V or III. On $\left(S N_{1}, H S N_{1}\right)$ we have a source and a saddle node characterized by a zero and a negative eigenvalue; on $\left(H S N_{1}, S N_{2}\right)$ and on $\left(S N_{4}, S N_{7}\right)$, a sink and a saddle-node characterized by a zero and a negative eigenvalue; on $\left(S N_{3}, S N_{4}\right)$ a sink and a saddle node characterized by a zero and a positive eigenvalue; on $\left(\mathrm{SN}_{4}, \mathrm{SN}_{5}, \mathrm{H}_{2}\right)$ two sinks, a saddle, and a saddle node characterized by a zero and a positive eigenvalue; on $\left(H_{2}, S N_{6}, H S N_{2}\right)$ two sinks, a saddle, and a saddle node characterized by a zero and a negative eigenvalue; and on $\left(H S N_{2}, S N_{4}\right)$ a sink, a saddle, a source, and a saddle node characterized by a zero and a negative eigenvalue.

2. On the curve $\left(H_{1}, H_{2}\right)$, denoted by $B_{H}$, one of the sinks becomes nonhyperbolic, having a pair of pure imaginary eigenvalues with a nonzero imaginary part. This is a Hopf bifurcation, and a limit cycle emerges, surrounding the considered fixed point. On $B_{H}$, this Hopf bifurcation is supercritical, leading to a stable limit cycle in domains II, IIIa, and VIIa.

3. At the point $\mathrm{H}_{2}$ (approximately (8.064.., 2.074..)) there are two sinks, a saddle, and a doubly degenerate fixed point with a zero eigenvalue of multiplicity two. This bifurcation is called a Bogdanov-Takens bifurcation.

4. We note that the phase portraits in domain III near $B_{H}$ and near $\left(S_{4}, S N_{3}\right)$ are not topologically equivalent since the former has a limit cycle while the latter does not. An additional bifurcation is present, causing the vanishing of the limit cycle through a saddle connection. We split III into two subdomains, IIIa and IIIb. We note $B_{S C 1}$ the bifurcation curve, which intersects $B_{S N 1}$ at $S C_{1}$ and $B_{S N 2}$ at $S C_{2}$. On $B_{S C 1}$ we have a homoclinic orbit of the hyperbolic saddle point; this saddle connection breaks the limit cycle as one crosses $B_{S C 1}$ from IIIa to IIIb. Moreover, on the curve $\left(S N_{1}, S C_{1}\right)$ and $\left(S C_{3}, S C_{2}\right)$ we have saddle node bifurcations on a limit cycle that exists in II, IIIa, and VIIa. 

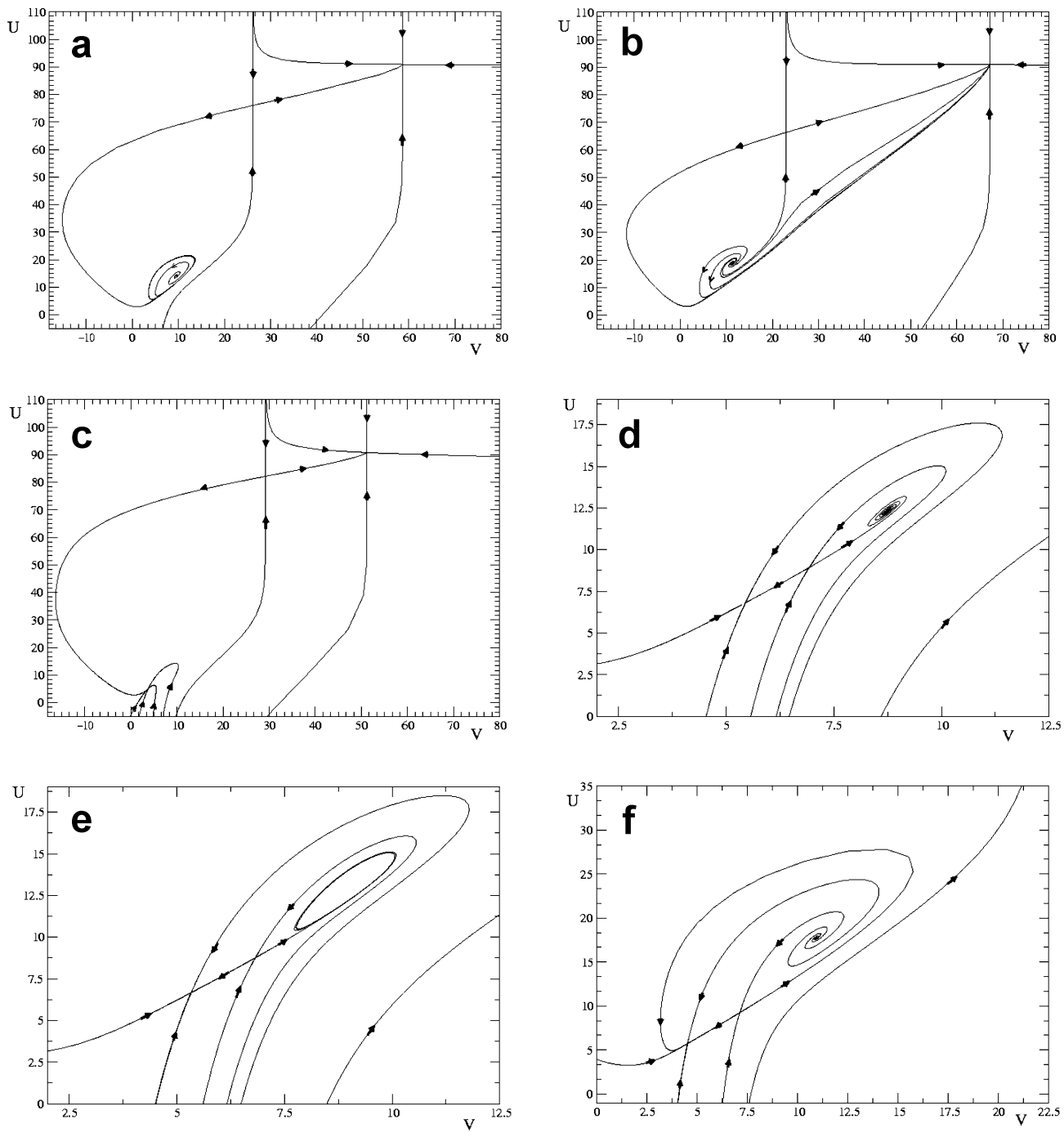

Fig. 4. The stable and unstable manifolds of the fixed points of Eq. 2 for $c_{e i}=10$ (cf. Fig. 3) and for different values of $\left(c_{e e}, c_{i e}\right)$. According to Fig. 3, parameters belong to the domain IIIa $(10,4.5)$ (Fig. 4a), IIIb $(10,3)$

(Fig. 4b), V (10,0.5) (Fig. 4c), VI $(9.2,3.75)$ (Fig. 4d), VIIa $(9.1,3.5)$

(Fig. 4e), VIIb $(8.5,2.2)$ (Fig. 4f). The other parameters are given in Fig. 3

5. As previously observed, an additional bifurcation curve is present in domain VII; therefore, we split this domain into VIIa and VIIb. The curve $B_{S C 2}$ is tangent to $B_{H}$ and $B_{S N 2}$ at $H_{2}$ and intersects $B_{S N 2}$ at $S C_{3}$. On $B_{S C 2}$ we have a homoclinic orbit of a hyperbolic saddle point; this saddle connection breaks the limit cycle surrounding the source as one crosses from VIIa to VIIb.

Note that in Sect. 3 we found a linear approximation of the $B_{S N 1}$ equation: $c_{i e}=\left(u_{e q}^{e e}-u_{\max }\right) /\left(u_{\max }-u_{e q}^{i e}\right) c_{e e}+$ $u_{\max } /\left(u_{e q}^{i e}-u_{\max }\right)$. Moreover the previous study gives the approximate position of the saddle points and the relative position of fixed points. One of the main differences with the piecewise linear case is that the resting state can disappear (through a saddle node bifurcation) or lose its stability (through a Hopf bifurcation).

Figure 4 gives the stable or unstable manifolds of the fixed points in the relevant domains IIIa, IIIb, V, VI, VIIa, and VIIb.

We now turn to Fig. 5, which corresponds to the second choice for the parameter $c_{e i}$, i.e., a moderate inhibitory scale. We split the $\left(c_{e e}, c_{i e}\right)$ space into six domains and number each domain according to the choice made in Fig. 3: (i) in domains I, II, and IV, we have one fixed point (a sink in I and IV and a source in II); (ii) in domain III, a sink, a saddle, and a source; and (iii) in domain $\mathrm{V}(\mathrm{Va} \cup \mathrm{Vb})$, two sinks and a saddle. However, in domain III, the location of the sink and the source is the reverse of that obtained in domain III of Fig. 3. The bifurcations obtained are:

1. On the curve $\left(S N_{1}, S N_{2}\right)$, denoted by $B_{S N 1}$, and on the curve $\left(S N_{3}, S N_{4}\right)$, denoted by $B_{S N 2}$, a saddle node bifurcation occurs. On $\left(S N_{1}, H S N_{1}\right)$ we have a source and a saddle node characterized by a zero and a negative eigenvalue; on $\left(H S N_{1}, S N_{2}\right)$ and on $\left(H_{2}, S N_{4}\right)$ we have a sink and a saddle node characterized by a zero and a negative eigenvalue; and on $\left(\mathrm{SN}_{3}, \mathrm{H}_{2}\right)$ we have a sink and a saddle node with a zero and a positive eigenvalue. Moreover, on the curve $\left(S N_{1}, H S N_{1}\right)$ the saddle node bifurcation occurs on the limit cycle.

2. On the curve $\left(H_{1}, H_{2}\right)$, denoted by $B_{H}$, one of the sinks becomes nonhyperbolic through a Hopf bifurcation, with a pair of eigenvalues with zero real part and nonzero imaginary part. On $\left(H_{1}, H S N_{1}\right)$ a stable limit 

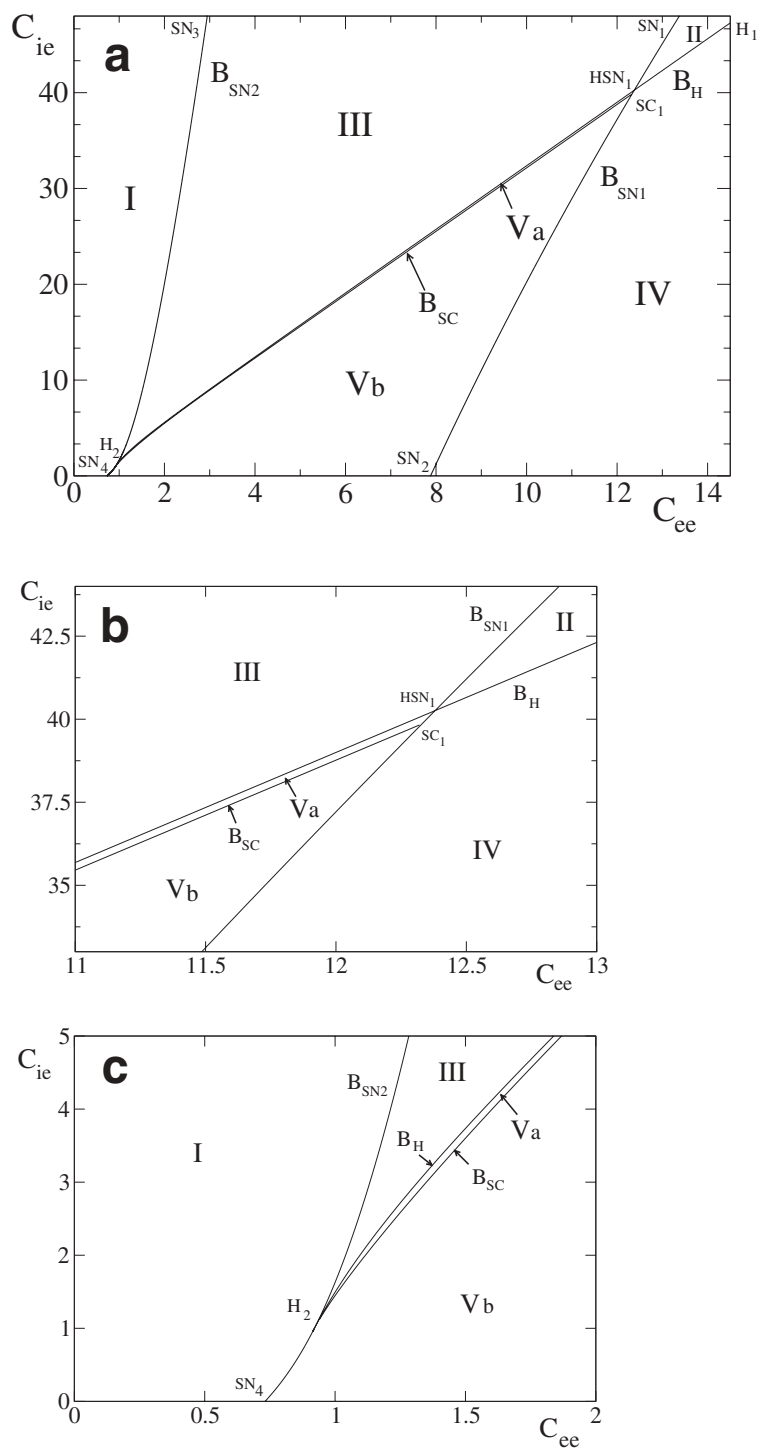

Fig. 5. The bifurcation diagram of Eq. 2 for $c_{e i}=0.4$; this value satisfies $u_{\min } /\left(u_{e q}^{e i}-u_{\min }\right)<c_{e i}<u_{\max } /\left(u_{e q}^{e i}-u_{\max }\right)$ and corresponds to one of the cases of proposition 3 . The other parameters are similar to those used in Fig. 3. Figure 5b and 5c are enlargements of Fig. 5a

cycle emerges, surrounding the considered fixed point (supercritical Hopf bifurcation). Conversely, on $\left(H S N_{1}, H_{2}\right)$ an unstable cycle occurs and the Hopf bifurcation is subcritical.

3. At the point $\mathrm{H}_{2}$ (approximately $(0.919 . ., 0.961 .$.$) )$ there are a sink and a doubly degenerate fixed point characterized by a zero eigenvalue of multiplicity two; this is a Bogdanov-Takens bifurcation.

4. There exists a fourth bifurcation curve, denoted by $B_{S C}$, which is tangent to $B_{H}$ and which intersects $B_{S N 2}$ at $H_{2}$. This curve splits domain $\mathrm{V}$ into domains $\mathrm{Va}$ and $\mathrm{Vb}$. On $B_{S C}$ we have a saddle node loop. An unstable limit cycle emerges as one enters domain Va.

The stable and unstable manifolds of fixed points in domains III, Va, and $\mathrm{Vb}$ are depicted in Fig. 6.

The analysis of the two bifurcation diagrams (Fig. 3 and Fig. 5) shows that there are values of parameters for which a stable limit cycle exists. This situation is related to the existence of a synchronous oscillatory activity in our network. Depending on the interaction strength, this cycle coexists with two or four fixed points that are stable nodes or saddle points. These oscillatory patterns exhibit long-lasting oscillations when parameters approach the saddle connection curve or the saddle node on a limit cycle curve. For other parameter values, the activity converges to an equilibrium corresponding to a resting steady state, when $v<u_{t h}$, or an excited state, when $v>u_{t h}$. When the resting steady state has a low activity, the system is said to be excitable, and, depending on the bifurcation that occurs, the excitability is referred as class I or II (Rinzel and Ermentrout 1989; Izhikevich 2000). As previously observed, large values of $c_{e i}$ (greater than $\left.u_{\max } /\left(u_{e q}^{e i}-u_{\max }\right)\right)$ give birth to nontrivial behavior: the bifurcation diagram is comprised of nine regions for $c_{e i}=10$ and seven regions for $c_{e i}=0.4$. An important qualitative difference is the destabilization of the excited state when $c_{e i}=0.4$ (Fig. 5, region III). This situation may be quite surprising since the inhibitory population is less excited, but it is explained by the relative difference between $c_{i e}$ and $c_{e e}$. Moreover, we observe that numerous stable patterns of synchronous activities are obtained for sufficiently strong excitatory interactions combined with a moderate inhibitory interaction (regions VI and VII).

Our study of the isolated neural oscillator does not provide a complete description of its bifurcation structure as $c_{e i}$ varies. An analysis of codimension three bifurcations as $c_{e i}$ continuously varies remains to be done. However, we have obtained a sequential description of the dynamical regimes for a high and a moderate value of $c_{e i}$.

\section{Excitation of neural oscillators}

We have studied the dynamical regimes of isolated populations. To go further in the analysis we consider the behavior of Eq. 2 under a constant excitatory input. This situation mimics the effects of a global homogeneous input on the synchronous activity of the network. We denote $i_{s}$ as the external input applied to the excitatory population. Equation 2 becomes

$$
\begin{aligned}
v^{\prime}(t)= & -v(t)+\left(c_{e e} \Phi(v(t))+i_{s}\right)\left(u_{e q}^{e e}-v(t)\right) \\
& +c_{i e} \Phi(u(t))\left(u_{e q}^{i e}-v(t)\right) \\
u^{\prime}(t)= & -u(t)+c_{e i} \Phi(v(t))\left(u_{e q}^{e i}-u(t)\right)
\end{aligned}
$$

The transformation: $\tilde{c}_{e e}=c_{e e}+i_{s}$ and $\tilde{\Phi}(x)=$ $\left(c_{e e} \Phi(x)+i_{s}\right) / \tilde{c}_{e e}$, yields for the excitatory population

$$
\begin{aligned}
v^{\prime}(t)= & -v(t)+\tilde{c}_{e e} \tilde{\Phi}(v(t))\left(u_{e q}^{e e}-v(t)\right) \\
& +c_{i e} \Phi(u(t))\left(u_{e q}^{i e}-v(t)\right)
\end{aligned}
$$

while the inhibitory one remains unchanged. Then the dynamical behavior of Eq. 4 is similar (but not identical) to those of an isolated region with a more reactive transfer function for the excitatory population since $\tilde{\Phi}(0)=\left(c_{e e} \Phi(0)+i_{s}\right)\left(c_{e e}+i_{s}\right)$. We consider the case 

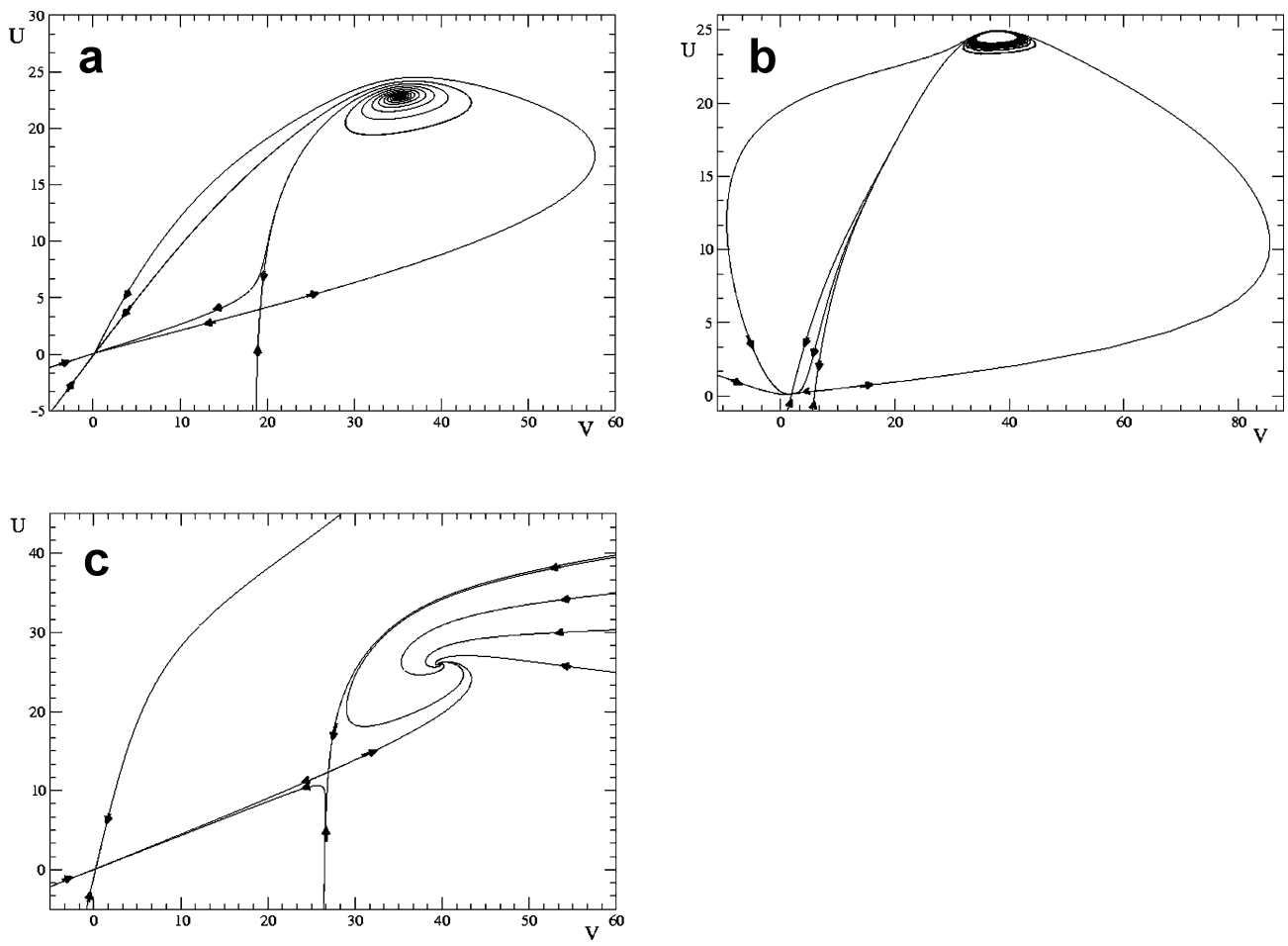

Fig. 6. The stable and unstable manifolds of the fixed points of Eq. 2 for $c_{e i}=0.4$ (cf. Fig. 5) and for different values of $\left(c_{e e}, c_{i e}\right)$. According to Fig. 5, $\left(c_{e e}, c_{i e}\right)$ belongs to III $(2.5,8)$ (Fig. 6a), Va $(11,35.6)$ (Fig. 6b), Vb $(1.1,1)$ (Fig. 6c). The other parameters are listed in Fig. 3. To be perfectly clear, all the manifolds are not represented

where parameters belong to domain I of the above bifurcation diagrams (Figs. 3 and 5). Then there exists a stable fixed point $\left(v_{0}, u_{0}\right)$, and the system is excitable. In Fig. 7, we draw the bifurcation diagram where $i_{s}$ is the bifurcation parameter. Specifically, we depicted the two cases, $c_{e i}=10$ and $c_{e i}=0.4$, which correspond to Fig. $7 \mathrm{a}$ and $7 \mathrm{~b}$, respectively. The curve labeled $C_{H}$ represents the $L^{2}$ norm of the cycle that emerges through a Hopf bifurcation, labeled $H_{1}, H_{2}$ for Fig. 7a and $H$ for Fig. 7b. Although the systems Eq. 2 and 4 are not identical, it is possible, through a homeomorphism, to identify the dynamical behavior obtained from Eq. 4 with those depicted in Figs. 4 and 6.

In Fig. $7 \mathrm{a}$, for $i_{s} \in\left[0, H_{1}\right]$, the system converges to the low-activity fixed point (domain I). At $i_{s}=H_{1}$ the fixed point loses its stability through a supercritical Hopf bifurcation, which gives birth to a stable limit cycle. For $i_{s} \in\left[H_{1}, H_{2}\right]$ the system oscillates; this situation corresponds to domain II. The limit cycle disappears at the second Hopf bifurcation point, $H_{2}$, but the activity remains low. At $i_{s}=L P_{1}$ a pair of fixed points appears, far from the low-level-activity fixed point. One of the fixed points is stable and corresponds to a high-level activity, and the other is a saddle point. At $i_{s}=L P_{2}$ the saddle point and the low-level-activity point merge, and for $i_{s}>L P_{2}$ the system is in high activity. As $i_{s}$ increases, the transition is summarized by the following graph: $I \rightarrow I I \rightarrow I \rightarrow V \rightarrow I V$.

We turn now to Fig. 7b. Three bifurcations occur as $i_{s}$ increases. At $i_{s}=L P_{1}$ a pair of fixed points appears far from the low-activity fixed point. One of the fixed points is a source and the other is a saddle point. At $i_{s}=L P_{2}$, the stable fixed point and the saddle point merge through a saddle node bifurcation. At point $H$ a supercritical Hopf bifurcation occurs, which gives birth to a stable limit cycle when $i_{s}$ is close to $H$ and $i_{s}<H$. As $i_{s}$ approaches $L P_{2}$, the period increases and becomes unbounded at $L P_{2}$. Therefore, the cycle disappears through a saddle node bifurcation on the limit cycle. As $i_{s}$ increases, the transition is summarized by the graph: $I \rightarrow I I I \rightarrow I I \rightarrow I V$.

We end this section with a remark that will be useful in the next section. A fixed point $\left(v_{0}\left(i_{s}\right), u_{0}\left(i_{s}\right)\right)$ is a solution of $l\left(v, i_{s}\right)=0$ with $l(x, y)=-x+\left(c_{e e} \Phi(x)\right.$ $+y)\left(u_{e q}^{e e}-x\right)+c_{i e} \Phi\left(c_{e i} u_{e q}^{e i} \Phi(x) /\left(1+c_{e i} \Phi(x)\right)\right)\left(u_{e q}^{i e}-x\right)$. We have:

$$
\begin{aligned}
v_{0}^{\prime}\left(i_{s}\right) & =-\frac{l_{x}\left(v_{0}\left(i_{s}\right), i_{s}\right)}{l_{y}\left(v_{0}\left(i_{s}\right), i_{s}\right)} \\
& =\frac{1}{\operatorname{det}(J)}\left(1+c_{e i} \Phi\left(v_{0}\left(i_{s}\right)\right)\right)\left(u_{e q}^{e e}-v_{0}\left(i_{s}\right)\right)
\end{aligned}
$$

Moreover, $u_{0}=c_{e i} u_{e q}^{e i} \Phi\left(v_{0}\right) /\left(1+c_{e i} \Phi\left(v_{0}\right)\right)$ is an increasing function of $v_{0}$. Then the two coordinates of a nonsaddle fixed point are increasing functions of $i_{s}$, while a saddle fixed point has coordinates that decrease with respect to $i_{s}$.

As we have shown, an increasing input yields a transition between the different dynamical regimes. We now use the specific transition low-activity stable state $\rightarrow$ oscillations $\rightarrow$ high-activity stable state to discuss the propagation of an activity in the network.

\subsection{Propagating activity}

In this section, we address the question of the propagation of an excitatory activity in a chain of connected neural 


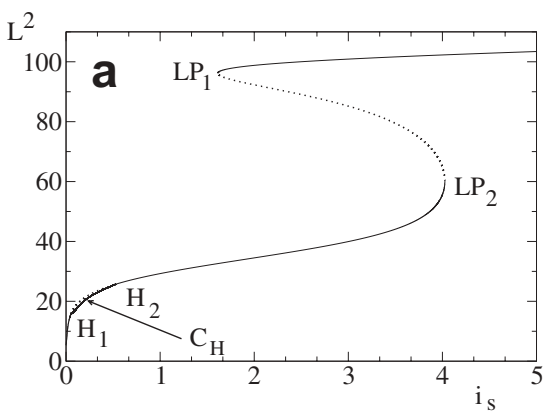

Fig. 7. The activities $(v, u)$ of Eq. 2 vs. $i_{s}$. Parameters $\left(c_{e e}, c_{i e}, c_{e i}\right)$ are equal to $(10,10,10)$ (Fig. 7a) and $(1,5,0.4)$ (Fig. 7b). Other parameters are given in Fig. 3. $L^{2}$ is the norm defined by: $1 / T \int_{0}^{T}\left(v^{2}(t)+u^{2}(t)\right) d t$, for a T-periodic solution and $u_{0}^{2}+v_{0}^{2}$ for a

oscillators. We consider a one-dimensional system with homogeneous feedforward connections between the excitatory pools. If we label the connection strength $c_{r}$, then the input current applied to the $k^{\text {th }}$ neural oscillator is given by $i_{s, k}=c_{r} \Phi\left(v_{k-1}\right)\left(u_{e q}^{e e}-v_{k}\right)$. Since an isolated neural oscillator stands for the synchronized activity of a population, our network mimics the activity of synchronous regions connected through a feedforward arrangement. The study of such a network appears to be relevant for biological models of cortical neural networks (Aertsen et al. 1998; Diesmann et al. 1999). It has been shown that the network supports traveling wave solutions like pulse waves and front waves (Tonnelier 2002). Here we focus our attention on traveling oscillatory activities driven by synaptic modifications.

We consider parameters such that an isolated region is in domain I (excitable regime) of the bifurcation diagrams depicted in Figs. 3 and 5. For $c_{r}$ close to 0, the implicit function theorem ensures the existence of a stable low-level activity for the network denoted by $\left\{\left(v_{0, k}, u_{0, k}\right)\right\}_{k=1 . . n}$. For the region $k$, the quantity $c_{r} \Phi\left(v_{0, k-1}\right)$ is similar to $i_{s}$ and from the previous study we have $v_{0, k}>v_{0, k-1}$ and $u_{0, k}>u_{0, k-1}, k=2 \ldots n$. Then, as $c_{r}$ increases, each neural oscillator covers the bifurcation diagram depicted in Fig. 7a or 7b. For a large network or for strong enough $c_{r}$, an index $k^{*}$ exists such that, for $k<k^{*}$, each neural oscillator is in a low-level activity. For $k>k^{*}$, neural oscillators present an oscillatory activity or are in a stable high level of activity. The boundary between high and low levels of activities is comprised of oscillatory neurons. This qualitative description is in agreement with numerical simulations and allows a distinction between the two media related to Fig. $7 \mathrm{a}$ and $7 \mathrm{~b}$, respectively. For a large excitatoryinhibitory interaction, i.e., large $c_{e i}$, oscillations appear through a Hopf bifurcation with a given frequency, while for small interaction strengths oscillations appear through a saddle node bifurcation with an arbitrarily small frequency. If we adopt a moderately slow increasing process on the synaptic strength $c_{r}$ such that $c_{r}$ is an increasing function of time, our network supports a traveling wave with an oscillatory wave front. Note that the propagation of the activity is induced by fixed point. The full lines indicate stable states and the dotted lines unstable ones. The branch of periodic solutions $\left(C_{H}\right)$ meets the branch of fixed points at (a) the two Hopf points $H_{1}, H_{2}$ and (b) at the saddle point $L P_{2}$ and the Hopf point $H$

increasing the connection strength between oscillators. The traveling wave happens only once and connects a stable low-level activity to a stable high-level activity. The oscillatory front presents two distinct mechanisms for the emerging frequency that could be used as a macroscopic feature of the media that allows one to distinguish between a large or small inhibitory scale.

\section{Discussion}

Two difficulties appear in modeling large and realistic neural networks: (i) the size of the neural population and (ii) the unknown connectivity of the network. We overcome this problem by considering regions that present synchronous activity. One expects that the synchronous activity of a neural network will play an important role in understanding more complex activities such as asynchronous states or spatiotemporal chaos.

Our macroscopic description of a synchronous region is given by a neural oscillator that mimics the activity of a pair of interacting excitatory and inhibitory neural populations. Parameters of our model stand for the extension of the synaptic interactions and the size of the population itself. An idealized nonlinearity (i.e., a piecewise linear transfer function) allowed us to obtain some analytical expressions for the bifurcations that may appear. For a regular function $\Phi$, numerical simulations enabled us to find all the dynamical behaviors through a bifurcation analysis. Using bifurcation diagrams (Figs. 3 and 5) one estimates the importance of the different scale parameters $\left(c_{e e}, c_{i e}, c_{e i}\right)$. Depending on the relative strength of these parameters, a large number of dynamical behaviors are encountered: oscillations, excitability, coexistence of fixed points, and a limit cycle. However, we only computed two projections of a general three-dimensional diagram. Further studies will be performed to reveal the codimension three bifurcations that yield the appearance of the new regions (regions VI, VIIa, and VIIb in Fig. 5) as $c_{e i}$ increases from a moderate value $\left(c_{e i}=0.4\right)$ to a high value $\left(c_{e i}=10\right)$.

We have found some properties already reported for the Wilson-Cowan oscillator (Borisyuk and Kirillov 
1992): (i) long-lasting oscillations, (ii) coexistence of stable fixed points and a stable limit cycle, and (iii) global bifurcations. Moreover, our macroscopic model has some basic properties of excitable cells already described by Rinzel and Ermentrout (1989). In particular, our model presents type I and type II mechanisms for excitability. Type II, where a single globally stable fixed point exists, is found in domain I of the two bifurcation diagrams (Figs. 3 and 5). Type I excitability, where three fixed points exist (a source, an asymptotically stable resting point, and a saddle point whose stable manifold acts as a threshold separatrix), is encountered in domain III of the bifurcation diagram depicted in Fig. 5 and is locally present in domain VII of Fig. 3.

From the study of an isolated neural oscillator we derived some properties of an excited neural oscillator. We showed that the features of the oscillations are monitored by the excitatory-inhibitory scale. Considering a feedforward arrangement of neural oscillators and adopting a synaptic modification, an oscillatory activity propagates in the network. The emerging frequency of the front could be used to predict the strength of the inhibitory interactions.

\section{Appendix}

We investigate the intersections of the nullclines and split the phase space into rectangular domains bounded by the following equations: $v=u_{\min }, v=u_{\max }, u=u_{\min }$, $u=u_{\max }$. We rigorously analyze the intersections of the nullclines and the stability of fixed points in the domains outside the square $\left[u_{\min }, u_{\max }\right] \times\left[u_{\min }, u_{\max }\right]$. In this square, intersection and stability analysis do not lead to tractable expressions.

1. For $v<u_{\min }$ : the $N L_{i}$ equation is given by $u=0$, and the $N L_{e}$ equation is $v=0$ (for $u<u_{\min }$ ). Then $(0,0)$ is the only fixed point in this region and the square centered at the origin with sides of length $2 u_{\min }$ belongs to the domain of attraction of $(0,0)$.

2. For $v>u_{\max }$ : the $N L_{i}$ equation is given by $u=c_{e i} u_{e q}^{e i} /$ $\left(1+c_{e i}\right)$ and $N L_{e}$ by:

$$
\begin{aligned}
\text { for } u<u_{\min }: v & =\frac{c_{e e} u_{e q}^{e e}}{1+c_{e e}} \\
\text { for } u>u_{\max }: v & =\frac{c_{e e} u_{e q}^{e e}+c_{i e} u_{e q}^{i e}}{1+c_{e e}+c_{i e}}
\end{aligned}
$$

These nullclines exist if their equations satisfy $v>u_{\max }$. In the domain $u_{\min }<u<u_{\max }, N L_{e}$ is a decreasing function. When $\left(c_{e e} u_{e q}^{e e}+c_{i e} u_{e q}^{i e}\right) /$ $\left(1+c_{e e}+c_{i e}\right)=u_{\max }$, and for $c_{e i} u_{e q}^{e i} /\left(1+c_{e i}\right)>u_{\max }$, a new fixed point $\left(u_{\max }, c_{e i} u_{e q}^{e i} /\left(1+c_{e i}\right)\right)$ appears. When $\left(c_{e e} u_{e q}^{e e}+c_{i e} u_{e q}^{i e}\right) /\left(1+c_{e e}+c_{i e}\right)>u_{\max }$, this new fixed point splits into two new ones. The stability of the new fixed points is given by studying the Jacobian matrix. Since the vector field is not differentiable at $u_{\max }$, we distinguish the Jacobian matrix on the right and on the left of $u_{\max }$ (denoted by $J^{+}$and $J^{-}$, respectively). We have

$$
\begin{aligned}
J^{+} & =\left(\begin{array}{cc}
-1-c_{e e}-c_{i e} & 0 \\
0 & -1-c_{e i}
\end{array}\right) \\
J^{-} & =\left(\begin{array}{cc}
-1-c_{i e}-c_{e e}+\lambda c_{e e}\left(u_{e q}^{e e}-u_{\max }\right) & 0 \\
\lambda \frac{c_{e i l}^{e i}}{1+c_{e i}} & -1-c_{e i}
\end{array}\right)
\end{aligned}
$$

Since we have $-1-c_{i e}-c_{e e}+\lambda c_{e e}\left(u_{e q}^{e e}-u_{\max }\right)>0$, the bifurcation gives rise to a stable node $\left(v>u_{\max }\right)$ and to a saddle $\left(v<u_{\max }\right)$, as expected from proposition 2. It is a saddle node bifurcation (Fig. 2).

3 . For $u_{\min }<v<u_{\max }: N L_{i}$ is a strictly increasing function. The $N L_{e}$ equation is given by solving a seconddegree polynomial:

$$
\begin{aligned}
& \text { for } u<u_{\min }, \\
& \begin{aligned}
P_{1}(v)= & v^{2}+\left(\frac{1}{\lambda c_{e e}}-u_{\min }-u_{e q}^{e e}\right) v+u_{e q}^{e e} u_{\min }=0 \\
P_{2}(v)= & v^{2}+\left(\frac{1+c_{i e}}{\lambda c_{e e}}-u_{\min }-u_{e q}^{e e}\right) v+u_{e q}^{e e} u_{\min } \\
& \quad-\frac{c_{i e} u_{e q}^{i e}}{\lambda c_{e e}}=0
\end{aligned}
\end{aligned}
$$

For $u_{\min }<u<u_{\max }, N L_{e}$ is strictly increasing, but precise conditions for its existence do not lead to a tractable expression. Note that for large $\lambda$, the $N L_{e}$ equation is: $v=u_{t h}+o(1)$, in both domains $u<u_{\text {min }}$ and $u>u_{\max }$. We do not perform the stability analysis of the fixed point in the domain $\left[u_{\min }, u_{\max }\right] \times$ $\left[u_{\min }, u_{\max }\right]$. Numerical simulations show that a Hopf bifurcation appears in this region.

\section{References}

Abbott L, Marder E (1998) Modeling small networks. In: Koch C, Segev I (eds) Methods in neuronal modeling, 2nd edn. MIT Press, Cambridge, MA, pp 135-169

Aertsen A, Diesmann M, Gewaltig MO (1998) Propagation of synchronous spiking activity in feedforward neural networks. J Physiol Paris 90: 243-247

Amari SI (1971) Characteristics of randomly connected thresholdelement networks and network systems. Proc IEEE 59: 35-47

Atiya A, Baldi P (1989) Oscillations and synchronizations in neural networks: an exploration of the labeling hypothesis. Int J Neural Sys 1: 103-124

Ballain T, Litaudon P, Martiel JL, Cattarelli M (1998) Role of the net architecture in piriform cortex activity: analysis by a mathematical model. Biol Cybern 79: 323-336

Borisyuk GN, Borisyuk RM, Khibnik AI, Roose D (1995) Dynamics and bifurcations of two coupled neural oscillators with different connection types. Bull Math Biol 57: 809-840

Borisyuk PM, Kirillov AB (1992) Bifurcation analysis of a neural network. Biol Cybern 66: 319-325

Bower JM, Beeman D (1994). The book of Genesis. Springer, Berlin Heidelberg New York

Chang HJ, Ghosh J, Liano K (1992) A macroscopic model of neural ensembles: learning-induced oscillations in a cell assembly. Int J Neural Sys 3: 179-198

Crook SM, Ermentrout GB, Vanier MC, Bower JM (1997) The role of axonal delay in the synchronization of networks of coupled cortical oscillators. J Comp Neurosci 4: 161-172

Destexhe A (1994) Oscillations, complex spatiotemporal behavior and information transport in networks of excitatory and inhibitory neurons. Phys Rev E 50: 1594-1606

Diesmann M, Gewaltig MO, Aertsen A (1999) Stable propagation of synchronous spiking in cortical neural networks. Nature 402: 529-533 
Doedel EJ (1981) AUTO, a program for the automatic bifurcation analysis of autonomous systems. Cong Numer 30: 265-384

Erdi P, Grobler T, Barna G, Kaski K (1993) Dynamics of the olfactory bulb: bifurcations, learning and memory. Biol Cybern 69: 57-66

FitzHugh R (1961) Impulses and physiological states in models of nerve membrane. Biophys J 1: 445-466

Guckenheimer J, Holmes P (1983) Nonlinear oscillations, dynamical systems, and bifurcations of vector fields. Springer, Berlin Heidelberg New York

Haberly LB, Price JL (1978) Association and commissural fiber system of the olfactory cortex of the rat. I. Systems originating in the piriform cortex and adjacent areas. J Comp Neurol 178: 711-740

Haberly LB, Price JL (1978) Association and commissural fiber system of the olfactory cortex of the rat. II. Systems originating from olfactory peduncle. J Comp Neurol 181: 781-808

Hodgkin AL, Huxley AF (1952) A quantitative description of membrane current and its application to conduction and excitation in nerve. J Physiol Lond 117: 500-544
Holmes PJ, Rand DA (1978) Bifurcations of the forced van der Pol oscillator. Q Appl Math 35: 495-509

Izhikevich YA (2000) Neural excitability, spiking, and bursting. Int J Bifurcation Chaos 10: 1171-1266

Kuznetsov YA (1995) Elements of applied bifurcation theory. Springer, Berlin Heidelberg New York

Rinzel J, Ermentrout GB (1989) Analysis of neural excitability and oscillations. In: Koch C, Segev I (eds) Methods in neuronal modeling, MIT Press, Cambridge, MA, pp 135-169

Rinzel J (1985) Excitation dynamics: insights from simplified membrane models. Federation Proc 44: 2944-2946

Tonnelier A (2002) Wave propagation in discrete media. J Math Biol 44: 87-105

Tonnelier A, Meignen S, Bosch H, Demongeot J (1999) Synchronization and desynchronization of neural oscillators. Neural Netw 12: 1213-1228

Wilson HR, Cowan JD (1972) Excitatory and inhibitory interaction in localized populations of model neurons. Biophys J $12: 1-24$ 